\title{
Huxley: Intelligent Book as Essentialist Artefact
}

\begin{abstract}
Huxley is a system that uses measurement of a user's focus and interest throughout the day in order to alter a 'smart-book' in their home space to make it more engaging. By tracking user interaction with the book over time, the home environment also learns to alter peripheral cues in imagery, lighting, and soundcontrolling the character of the space and the visual salience of the book - in order to encourage its use. Huxley is an environment that is informed about user goals and works to encourage better decisions and deeper focus in line with those intentions. It is an artefact of an essentialist, attention-centered design philosophy.
\end{abstract}

\section{Keywords}

Attention, Salience, Smart Home, Persuasion, Cognitive Affordance

\section{Introduction}

A decade ago Nicholas Carr first raised the alarm about negative impacts of the information age on human cognition and phenomenology. [1] It is now a chorus in the popular tech literature-our focus and concentration has been hijacked by our information and communication technology (ICT). Carr argues that the rapid, non-linear navigation of a hyperlinked internet has already predisposed us away from deep, focused reflection on singular topics. Ad-driven business models - which necessitate addictive design patterns and a diversion of users away from their intentintensifies a trend towards fractured attention that is native to the medium itself.

Unfortunately, the research bears this out. Most websites are visited for less than 15 seconds; all websites average less than a minute. [2] Moreover, hyperlinked content detracts from learning outcomes while increasing interaction time. [3] Smartphone research is more directly disheartening-Ward et. al.'s 2017 work [4] showed that having one's phone in the visual periphery significantly reduces one's available cognitive capacity for other tasks.

Against the backdrop of behavioural economics, we can frame the internet and the smartphone another waytheir structures impose a state of constant decisionmaking on the user. These designs force a regular reevaluation of the task at hand; with instant and limitless access, they also prevent us from full commitment to any one task. We know from the work of psychologist Barry Schwartz that too many choices lead to paradoxical dissatisfaction. [5] The ability to renege on our selections also leads to greater unhappiness, despite our tendency to keep options available. [6] 
How should this knowledge impact future design? While there are many open questions about the nature of the relationships between an artefact's symbolic information, conceptual model, [7] and its cognitive effects, it is clear that we certainly should avoid conceptual models and addictive design practices that are linked to cognitive overhead, and likely should avoid the corresponding symbolic information that has been rigorously paired with fractured attention over the last decade. More research needs to be done to answer many important questions:

- How tightly coupled are conceptual models and cognitive load? Is the relationship binary or continuous? (If your phone decreases your focus, will a similar phone? Any phone?)

- Have we learned a direct association between symbolic information and cognitive state? Do new conceptual models alter it? (Does a 'phone form' induce high cognitive load, even with no phone functionality? Is this reversible?)

- What aspects of these devices are most damaging to focus? (How much does the type and nature of a device's functionality versus addictive design choices like variable reward structures, badges, and infinite scroll contribute to cognitive load?)

In the spirit of 'Calm Technology', [8] Huxley is designed against the backdrop of these provocations, both as a tool for inquiry and as an example of attentionaware design. Other theories of design offer notions of psychological pairing between an object's sensory features and a user's emotional response to it; [9] we submit this idea logically extends to the user's resting attentional demand when using the object as well. We believe the psychology supports a move toward essentialism [10] in design-environments and artefacts whose choice architecture promotes fewer, longer, deeply engaged experiences in line with a user's priorities for themselves.

There is a rich confluence of cause-and-effect to disentangle given the current state of screen-based, multifunction artefacts. We hope Huxley will spark fruitful conversation about the cultural and aesthetic implications of the Attention Economy's addictive design tradition and move us towards an empirical design language based on behavioural economics and cognitive models of deep engagement.

\section{Huxley}

The Huxley system is composed of three main parts: a chrome extension to track focus and interest during the day, the smart book artefact itself, and a peripheral lighting and video system to alter the affective quality of the reading environment and book salience.

\subsection{Interest Tracking}

The first part of Huxley is a chrome extension that runs in the browser on the user's primary work computer, collecting and storing data including the number of tabs and windows open, the websites visited, the number of task switches, and overall active and idle behaviour. This information is processed to give a measure of focus, the duration worked, and the topics the user is engaging with based on Google queries, time on 'timewasting' or 'productive' domains, task switching, and other browsing behaviour.

\subsection{Smart Book}

Huxley (Fig. 1) is a book with three e-ink displays, a single button, and a charging USB port. It behaves like a book-its spine and cover display the title and cover of the book contained within, and once opened the
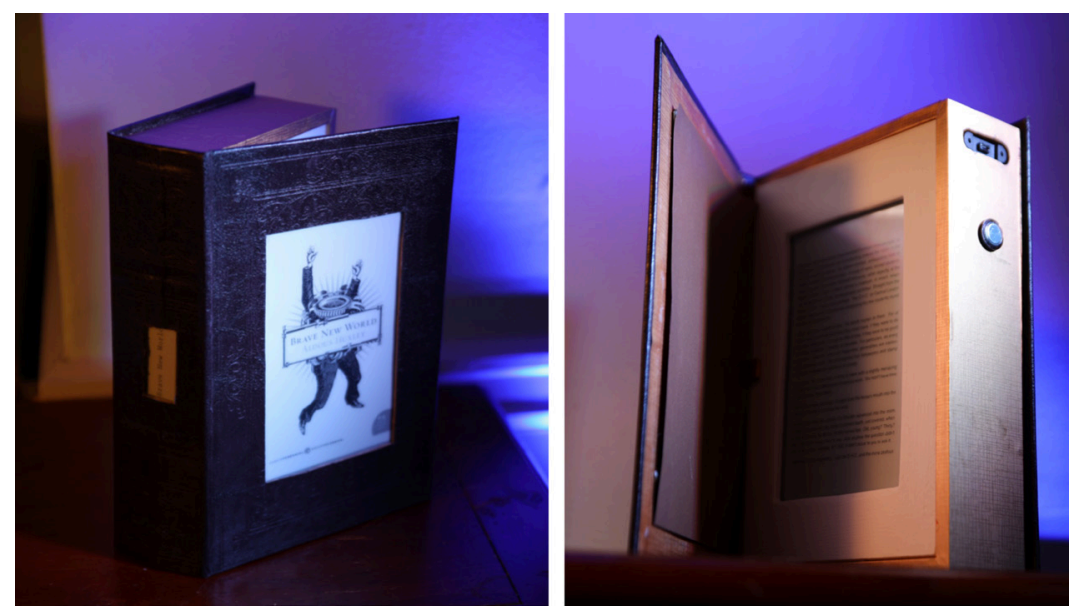

Fig. 1. The Huxley Smart Book, showing updating e-ink displays on the cover, spine, and inside. A USB port on top is available for charging, one button is available for turning pages. Huxley only updates when the user is away and the current book has been completed or ignored. Its selection is based on the measured interests and affective state of the user at work. 
internal e-ink page can be turned with a button press. There are no indicators of battery state or wireless connectivity, no menus, and no way to change the book contained within.

Instead, Huxley broadcasts an API over the local network-once loaded with PDF/EPUB files, it can be directed to become a random selection (favoring new books) or become a relevant book based on provided queries (using Doc2 Vec embeddings as a similarity metric).

Huxley was created with a few motivating principles in mind:

Minimized Choice. Good technology helps curate our world and eliminate the ill effects of the paradox of choice when we're trying to focus. There is no ability with Huxley to summon any book in the universe, and no leaving the option in front of you behind.

Permanence. Huxley only switches when the user is away. The interaction is designed to make it feel like a single, permanent object that doesn't have the ability to access any external information.

Simplicity. The conceptual model is very simple-- it works like a book. It doesn't have WIFI or battery indicators. The affordances are equally simple-one button, one possible interaction.

Evocative Aesthetics. Huxley evokes the conceptual model of a book without false affordances (it's clear there are no pages to turn). This helps frame the interaction as 'book-like' instead of 'screen-like'.

Increased Cost. Many of our services allow us ephemeral access to unlimited media. The 'zero-cost' model (time, space, money, and effort) disincentivized attribution of value to any included content. Huxley takes up space in the home, a physical cost that ascribes worth to its content and incentivizes focused engagement.

\subsection{Peripheral Guiding and Intelligence}

Users prefer different lighting for different tasks; [11] we hope that priming the mood also encourages the associated activity. Increasing a behaviour like reading usually involves more frequent 'triggers' to perform the action. [12] By manipulating book salience, we aim to draw the user's eye to the book more frequently - the more they notice and consider it, the more likely they are to engage with it.

Lighting, visual imagery, and book spotlighting are controlled using a custom video looping/DMX lighting control server running on a Raspberry Pi. Though the system is capable of a full range of expression, for simplicity we've devised two basic conditions (Fig. 2). The system uses a running average to bucket focus and time worked from the interest tracker into four categories (i.e. high focus, long day). Each of these categories is treated separately with a Bayesian Bandit optimization over lighting choice and book relatedness to work queries. We seed the system with common sense priors - for example, we expect long focused days to benefit from a calming off-topic book, short focused days to benefit from a high-energy related book, etc. By monitoring page turns, we update our model each day to choose the best lighting and book pairings to encourage use for a given user state.

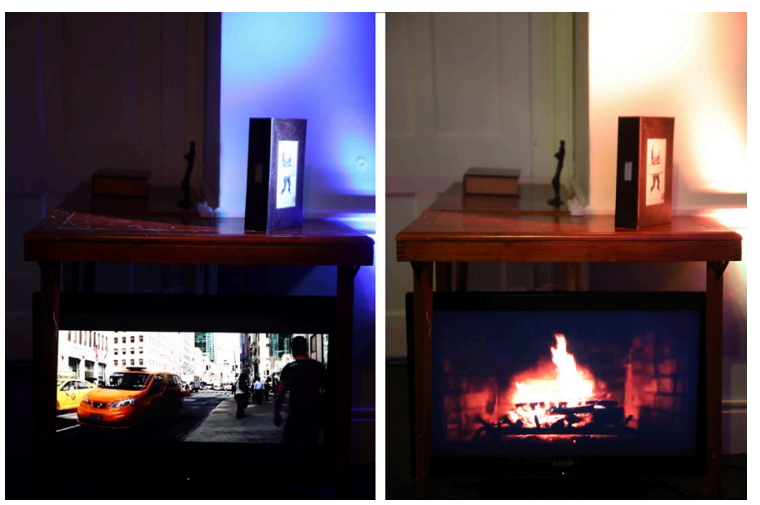

Fig. 2. Left, a high-energy scene of a city, with blue lights and a white spotlight on the book. Right, a warm scene with a fireplace and without book spotlighting. Mood and book salience are selected based on the Focus Tracking extension.

\section{Discussion and Next Steps}

Huxley is an adaptive system that nudges you towards a deeply engaged reading experience through peripheral cues, content selection, and artefact design. Its design principles are derived from essentialism in reaction to the current paradigm of attention hacking. We plan to study this interaction and quantify the its effect on the number and depth of book interactions compared with books, e-readers, and iPads in situ. We hope to 
disentangle the influence of conceptual models and symbolic information on deep engagement-mostly learned, modern associations with ramifications for future design.

We also plan to introduce eye tracking to explicitly quantify saliency's effect on behaviour and attempt to quantify the benefits of peripheral cues, alongside richer measures and models of user state. This will pave the way for more accurate and sophisticated analysis of dynamic causal intervention, and lead to a more effective overall system.

\section{Conclusion}

How do we design systems that increase our likelihood of doing the things we aspire to more frequently and with deeper focus? Can we marry the best of iPads to books, Spotify to records, or email to letters? Huxley was created with an understanding of the inherited problems of attention economy design, with a goal of promoting deep engagement. It attempts to preserves the value of connected information technology, and behavioral nudging without the negative cognitive impacts. It is an artefact of a design philosophy built for focus.

Huxley strives to feel like a close friend selected a single book for you every night after work; not an open portal to limitless options. We hope more technology moves towards essentialism and away from the fractured attention characteristic of the modern technology landscape.

\section{References}

1. Carr, N. (2011). The shallows: What the Internet is doing to our brains. WW Norton \& Company.

2. Liu, C., White, R. W., \& Dumais, S. (2010, July).

Understanding web browsing behaviors through Weibull

analysis of dwell time. In Proceedings of the 33rd

international ACM SIGIR conference on Research and

development in information retrieval (pp. 379-386). ACM.

3. Niederhauser, D. S., Reynolds, R. E., Salmen, D. J., \& Skolmoski, P. (2000). The influence of cognitive load on learning from hypertext. Journal of educational computing research, 23(3), 237-255.
4. Ward, A. F., Duke, K., Gneezy, A., \& Bos, M. W. (2017). Brain drain: the mere presence of one's own smartphone reduces available cognitive capacity. Journal of the Association for Consumer Research, 2(2), 140-154.

5. Schwartz, B. (2004). The paradox of choice: Why more is less (Vol. 6). New York: HarperCollins.

6. Gilbert, D. (2009). Stumbling on happiness. Vintage Canada.

7. Norman, D. A. (1999). Affordance, conventions, and design. interactions, 6(3), 38-43.

8. Weiser, M., \& Brown, J. S. (1996). Designing calm technology. PowerGrid Journal, 1(1), 75-85.

9. Colombo, S. (2016). Dynamic Products: Shaping Information to Engage and Persuade. Springer.

10. McKeown, G. (2014). Essentialism: The disciplined pursuit of less. Currency.

11. Zhao, N., Azaria, A., \& Paradiso, J. A. (2017). Mediated Atmospheres: A Multimodal Mediated Work Environment. Proceedings of the ACM on Interactive, Mobile, Wearable and Ubiquitous Technologies, 1(2), 31.

12. Fogg, B. J. (2009, April). A behavior model for persuasive design. In Proceedings of the 4th international Conference on Persuasive Technology (p. 40). ACM. 